\title{
Positive Periodic Solutions of a Periodic Discrete Competitive System Subject to Feedback Controls
}

\author{
Ronghua Tan, ${ }^{1,2}$ Zuxiong Li, $^{2}$ Qinglong Wang, ${ }^{2}$ and Zhijun Liu ${ }^{1,2}$ \\ ${ }^{1}$ Key Laboratory of Biologic Resources Protection and Utilization of Hubei Province, Hubei University for Nationalities, Enshi, \\ Hubei 445000, China \\ ${ }^{2}$ Department of Mathematics, Hubei University for Nationalities, Enshi, Hubei 445000, China
}

Correspondence should be addressed to Zhijun Liu; zhijun_liu47@hotmail.com

Received 13 February 2014; Revised 12 June 2014; Accepted 16 June 2014; Published 30 June 2014

Academic Editor: Shiping Lu

Copyright (C) 2014 Ronghua Tan et al. This is an open access article distributed under the Creative Commons Attribution License, which permits unrestricted use, distribution, and reproduction in any medium, provided the original work is properly cited.

\begin{abstract}
Species living in a fluctuating medium and human exploitation activities might result in the duration of continuous changes. Such changes can be well-approximated as feedback controls. In this contribution a periodic discrete competitive system subject to feedback controls is proposed. By using the methods of discrete inequality, fixed point theorem, and analysis techniques, a good understanding of the existence and global asymptotic stability of positive periodic solutions is obtained. Some numerical investigations are provided to verify our analytical results.
\end{abstract}

\section{Introduction}

Many mathematical models about bioecology play important roles for researchers to realize the interactions of ecological species. There has been increasing interest in studying the dynamical behaviors such as stability, permanence, and periodicity of competitive systems (see [1-5]). Recently, motivated by an autonomous competitive model of [6] in [7] we introduced a corresponding nonautonomous version

$$
\begin{gathered}
y_{1}^{\prime}(t)=y_{1}(t)\left[a_{1}(t)-b_{1}(t) y_{1}(t)-c_{1}(t) y_{2}(t)\right. \\
\left.-d_{1}(t) y_{1}^{2}(t)\right], \\
y_{2}^{\prime}(t)=y_{2}(t)\left[a_{2}(t)-b_{2}(t) y_{2}(t)-c_{2}(t) y_{1}(t)\right. \\
\left.-d_{2}(t) y_{2}^{2}(t)\right],
\end{gathered}
$$

where $y_{1}(t)$ and $y_{2}(t)$ can be interpreted as the density of two competing species at time $t$, respectively. $a_{i}(t)$ stand for the growth rates of species, $b_{i}(t)$ and $d_{i}(t)$ represent the effects of intraspecific competition, and $c_{i}(t)$ are the rates of interspecific competition. Furthermore, we took the influence of almost periodic environment and impulsive perturbations into account and established sufficient conditions for the uniformly asymptotic stability of a unique positive almost periodic solution for the above system (for details see [7]).

Note that ecosystems in the real world are often disturbed by outside continuous forces which can lead to changes in biological coefficients such as survival rates. In the language of control, we call the disturbance functions as feedback control variables. Many good results on this direction are deliberated (see [8-12]) and some similar works on the topic have been done (see $[13,14])$. This paper is concerned with a discrete model and is a continuation of the work in [7]; in this contribution we search for certain schemes (such as harvesting procedure) to ensure the system coexists under appropriate conditions. For this reason, we consider a discrete version which corresponds to differential version (i.e., system (1)), meanwhile, replacing abrupt external perturbations (impulses) in [7] by continuous external perturbations (feedback control variables), a nonautonomous discrete controlled system can be described as follows:

$$
\begin{aligned}
y_{1}(k+1)=y_{1}(k) \exp [ & a_{1}(k)-b_{1}(k) y_{1}(k)-c_{1}(k) y_{2}(k) \\
& \left.-d_{1}(k) y_{1}^{2}(k)-e_{1}(k) v_{1}(k)\right], \\
y_{2}(k+1)=y_{2}(k) \exp [ & a_{2}(k)-b_{2}(k) y_{2}(k)-c_{2}(k) y_{1}(k) \\
- & \left.d_{2}(k) y_{2}^{2}(k)-e_{2}(k) v_{2}(k)\right],
\end{aligned}
$$




$$
\begin{aligned}
\Delta v_{1} & =v_{1}(k+1)-v_{1}(k) \\
& =h_{1}(k)-f_{1}(k) v_{1}(k)+g_{1}(k) y_{1}(k), \\
\Delta v_{2} & =v_{2}(k+1)-v_{2}(k) \\
& =h_{2}(k)-f_{2}(k) v_{2}(k)+g_{2}(k) y_{2}(k), \quad k \in \mathbb{Z}^{+} .
\end{aligned}
$$

Here $y_{i}(0)>0, v_{i}(0)>0 . \Delta v_{i}(k)$ are the first-order forward difference operators, $y_{i}(k)$ stand for the densities of species $y_{i}$ at the $k$ th generation, $a_{i}(k)$ represent the natural growth rates of species $y_{i}$ at the $k$ th generation, $b_{i}(k)$ and $d_{i}(k)$ stand for the intraspecific effects of the $k$ th generation of species $y_{i}$ on own population, and $c_{i}(k)$ measure the interspecific effects of the $k$ th generation of species $y_{j}$ on species $y_{i}$. The coefficients $\left\{a_{i}(k)\right\},\left\{b_{i}(k)\right\},\left\{c_{i}(k)\right\},\left\{d_{i}(k)\right\},\left\{e_{i}(k)\right\},\left\{f_{i}(k)\right\},\left\{g_{i}(k)\right\}$, and $\left\{h_{i}(k)\right\}$ are $T$-periodic sequences with $0<f_{i}(k)<1, i, j=$ 1,$2 ; i \neq j$. $\mathbb{Z}^{+}$is the set of nonnegative integers.

It is well known that the discrete models governed by difference equations may be more appropriate than the continuous ones when populations have a short life expectancy or nonoverlapping generations. Also, discrete models can provide efficient computational methods of continuous models for numerical simulations. A very important ecological problem associated with the study of multispecies population interaction in a periodic environment is the positive periodic solution which plays the role played by the equilibrium of the autonomous model. In this paper, we will discuss the above discrete system (2) and focus on the existence and stability of positive periodic solution of system (2). To the best of our knowledge, there are few published papers concerning system (2).

The rest structure of this paper is as follows. In the next section, we establish sufficient conditions for the existence of positive periodic solutions. In Section 3, we further discuss the global asymptotic stability of positive periodic solutions. In Section 4, we carry out an example and its numerical simulations to substantiate our theoretical results. To simplify the reading of paper, we give the proofs of lemmas and theorems in appendices.

\section{Existence}

In this section, we establish sufficient conditions for the existence of positive periodic solutions of system (2). To do this, we first give two preliminary lemmas. For convenience, given a bounded sequence $\{f(k)\}$, let $f^{U}$ and $f^{L}$ be defined as $f^{U}=\sup _{k \in \mathbb{Z}^{+}} f(k), f^{L}=\inf _{k \in \mathbb{Z}^{+}} f(k)$, and

$$
\begin{gathered}
M_{i}=\frac{\exp \left(a_{i}^{U}-1\right)}{b_{i}^{L}}, \quad N_{i}=\frac{h_{i}^{U}+g_{i}^{U} M_{i}}{f_{i}^{L}}, \\
m_{i}=\frac{a_{i}^{L}-c_{i}^{U} M_{j}-d_{i}^{U} M_{i}^{2}-e_{i}^{U} N_{i}}{b_{i}^{U}} \\
\times \exp \left(a_{i}^{L}-b_{i}^{U} M_{i}-c_{i}^{U} M_{j}-d_{i}^{U} M_{i}^{2}-e_{i}^{U} N_{i}\right), \\
n_{i}=\frac{\left(h_{i}^{L}+g_{i}^{L} m_{i}\right)}{f_{i}^{U}}, \quad i \neq j ; i, j=1,2 .
\end{gathered}
$$

Lemma 1. Every positive solution $\left\{y_{1}(k), y_{2}(k), v_{1}(k), v_{2}(k)\right\}$ of system (2) satisfies $\limsup _{k \rightarrow+\infty} y_{i}(k) \leq M_{i}$ and $\limsup _{k \rightarrow+\infty} v_{i}(k) \leq N_{i}, i=1,2$.

The proof of Lemma 1 is given in Appendix A.

Lemma 2. Assuming that

$$
\begin{aligned}
& a_{1}^{L}-c_{1}^{U} M_{2}-d_{1}^{U} M_{1}^{2}-e_{1}^{U} N_{1}>0, \\
& a_{2}^{L}-c_{2}^{U} M_{1}-d_{2}^{U} M_{2}^{2}-e_{2}^{U} N_{2}>0
\end{aligned}
$$

hold, then every positive solution $\left\{y_{1}(k), y_{2}(k), v_{1}(k), v_{2}(k)\right\}$ of system (2) satisfies $\liminf _{k \rightarrow+\infty} y_{i}(k) \geq m_{i}$ and $\liminf _{k \rightarrow+\infty} v_{i}(k) \geq n_{i}, \quad i=1,2$.

The proof of Lemma 2 is given in Appendix B.

Theorem 3. If the inequalities in (4) hold, then system (2) has at least one strictly positive T-periodic solution.

The proof of Theorem 3 is given in Appendix C.

\section{Global Asymptotic Stability}

In this section, we further investigate the stability of positive periodic solutions of system (2). Denote

$$
\begin{gathered}
\rho_{1}=\max \left\{\left|1-b_{1}^{L} m_{1}-2 d_{1}^{L} m_{1}^{2}\right|,\left|1-b_{1}^{U} M_{1}-2 d_{1}^{U} M_{1}^{2}\right|\right\} \\
+c_{1}^{U} M_{2}+e_{1}^{U}, \\
\rho_{2}=\max \left\{\left|1-b_{2}^{L} m_{2}-2 d_{2}^{L} m_{2}^{2}\right|,\left|1-b_{2}^{U} M_{2}-2 d_{2}^{U} M_{2}^{2}\right|\right\} \\
+c_{2}^{U} M_{1}+e_{2}^{U}, \\
\rho_{3}=1-f_{1}^{L}+g_{1}^{U} M_{1}, \\
\rho_{4}=1-f_{2}^{L}+g_{2}^{U} M_{2} .
\end{gathered}
$$

Theorem 4. In addition to (4), further suppose that $\rho_{i}<1$, $i=1,2,3,4$. Then the positive periodic solution of system (2) is globally asymptotically stable.

The proof of Theorem 4 is given in Appendix D.

\section{An Example}

In this section we give a numerical example and its corresponding simulations. 
Example 1. Consider a discrete competitive system with feedback controls

$$
\begin{aligned}
& y_{1}(k+1)=y_{1}(k) \exp [ 0.98+0.003 \sin (k \pi) \\
&-(0.85-0.002 \cos (k \pi)) y_{1}(k) \\
&-(0.002+0.0001 \sin (k \pi)) y_{2}(k) \\
&-(0.004-0.0002 \cos (k \pi)) y_{1}^{2}(k) \\
&\left.-(0.003+0.0001 \sin (k \pi)) v_{1}(k)\right], \\
& y_{2}(k+1)=y_{2}(k) \exp [1.00-0.002 \sin (k \pi) \\
&-(0.92+0.001 \cos (k \pi)) y_{2}(k) \\
&-(0.003+0.0001 \cos (k \pi)) y_{1}(k) \\
&-(0.005+0.0003 \sin (k \pi)) y_{2}^{2}(k) \\
&\left.-(0.002+0.0002 \sin (k \pi)) v_{2}(k)\right],
\end{aligned}
$$

$\Delta v_{1}(k)=0.005+0.0002 \sin (k \pi)$

$$
\begin{aligned}
& -(0.95+0.003 \cos (k \pi)) v_{1}(k) \\
& +(0.006+0.0001 \sin (k \pi)) y_{1}(k),
\end{aligned}
$$

$$
\begin{aligned}
\Delta v_{2}(k)= & 0.004+0.0001 \sin (k \pi) \\
& -(0.98+0.002 \sin (k \pi)) v_{2}(k) \\
& +(0.003+0.0002 \cos (k \pi)) y_{2}(k) .
\end{aligned}
$$

A computation shows that

$$
\begin{array}{ccc}
a_{1}^{L}=0.977, & a_{1}^{U}=0.983, & b_{1}^{L}=0.848, \\
b_{1}^{U}=0.852, & c_{1}^{L}=0.0019, & c_{1}^{U}=0.0021, \\
d_{1}^{L}=0.0038, & d_{1}^{U}=0.0042, & e_{1}^{L}=0.0029, \\
e_{1}^{U}=0.0031, & f_{1}^{L}=0.947, & f_{1}^{U}=0.953, \\
g_{1}^{L}=0.0059, & g_{1}^{U}=0.0061, & h_{1}^{L}=0.0048, \\
h_{1}^{U}=0.0052, & a_{2}^{L}=0.998, & a_{2}^{U}=1.002, \\
b_{2}^{L}=0.919, & b_{2}^{U}=0.921, & c_{2}^{L}=0.0029, \\
c_{2}^{U}=0.0031, & d_{2}^{L}=0.0047, & d_{2}^{U}=0.0053, \\
e_{2}^{L}=0.0018, & e_{2}^{U}=0.0022, & f_{2}^{L}=0.978, \\
f_{2}^{U}=0.982, & g_{2}^{L}=0.0028, & g_{2}^{U}=0.0032, \\
& h_{2}^{L}=0.0039, \quad h_{2}^{U}=0.0041 .
\end{array}
$$

Furthermore, it follows from (3) that

$$
\begin{aligned}
& M_{1} \approx 1.1594, \quad M_{2} \approx 1.0903, \\
& N_{1} \approx 0.0130, \quad N_{2} \approx 0.0078, \\
& m_{1} \approx 1.1162, \quad m_{2} \approx 1.0557, \\
& n_{1} \approx 0.0119, \quad n_{2} \approx 0.0070, \\
& a_{1}^{L}-c_{1}^{U} M_{2}-d_{1}^{U} M_{1}^{2}-e_{1}^{U} N_{1} \approx 0.9690>0, \\
& a_{2}^{L}-c_{2}^{U} M_{1}-d_{2}^{U} M_{2}^{2}-e_{2}^{U} N_{2} \approx 0.9881>0 .
\end{aligned}
$$

Obviously, the assumptions in (4) are satisfied, and moreover, one has

$$
\begin{aligned}
\rho_{1}= & \max \left\{\left|1-b_{1}^{L} m_{1}-2 d_{1}^{L} m_{1}^{2}\right|,\left|1-b_{1}^{U} M_{1}-2 d_{1}^{U} M_{1}^{2}\right|\right\} \\
& +c_{1}^{U} M_{2}+e_{1}^{U} \approx 0.0494<1, \\
\rho_{2}= & \max \left\{\left|1-b_{2}^{L} m_{2}-2 d_{2}^{L} m_{2}^{2}\right|,\left|1-b_{2}^{U} M_{2}-2 d_{2}^{U} M_{2}^{2}\right|\right\} \\
+ & c_{2}^{U} M_{1}+e_{2}^{U} \approx 0.0251<1, \\
& \rho_{3}=1-f_{1}^{L}+g_{1}^{U} M_{1} \approx 0.0601<1, \\
& \rho_{4}=1-f_{2}^{L}+g_{2}^{U} M_{2} \approx 0.0255<1,
\end{aligned}
$$

so the assumptions of Theorem 4 are also satisfied. Thus, there exists a globally asymptotically stable positive periodic solution of system (6). Figure 1 shows that system (6) exists a positive periodic solution $\left(y_{1}^{*}(k), y_{2}^{*}(k), v_{1}^{*}(k), v_{2}^{*}(k)\right)$, and the two-dimensional phase portrait of periodic system (6) is displayed in Figure 2. From Figure 3, we can see that any positive solution $\left(y_{1}(k), y_{2}(k), v_{1}(k), v_{2}(k)\right)$ tends to the periodic solution $\left(y_{1}^{*}(k), y_{2}^{*}(k), v_{1}^{*}(k), v_{2}^{*}(k)\right)$, and the twodimensional phase portrait reflects the fact in Figure 4.

\section{Appendices}

\section{A. Proof of Lemma 1}

We consider the following Cases I and II to show that

$$
\limsup _{k \rightarrow+\infty} y_{i}(k) \leq M_{i}, \quad i=1,2 .
$$

Case I. Suppose that there is a $k_{0} \in \mathbb{Z}^{+}$such that $y_{1}\left(k_{0}\right) \leq$ $y_{1}\left(k_{0}+1\right)$, it follows from the first equation of system (2) that

$$
\begin{aligned}
& y_{1}\left(k_{0}+1\right) \\
& =y_{1}\left(k_{0}\right) \exp \left[a_{1}\left(k_{0}\right)-b_{1}\left(k_{0}\right) y_{1}\left(k_{0}\right)-c_{1}\left(k_{0}\right) y_{2}\left(k_{0}\right)\right. \\
& \left.\quad-d_{1}\left(k_{0}\right) y_{1}^{2}\left(k_{0}\right)-e_{1}\left(k_{0}\right) v_{1}\left(k_{0}\right)\right] \\
& \leq y_{1}\left(k_{0}\right) \exp \left[a_{1}^{U}-b_{1}^{L} y_{1}\left(k_{0}\right)\right] \\
& \leq \frac{\exp \left(a_{1}^{U}-1\right)}{b_{1}^{L}}=M_{1} .
\end{aligned}
$$




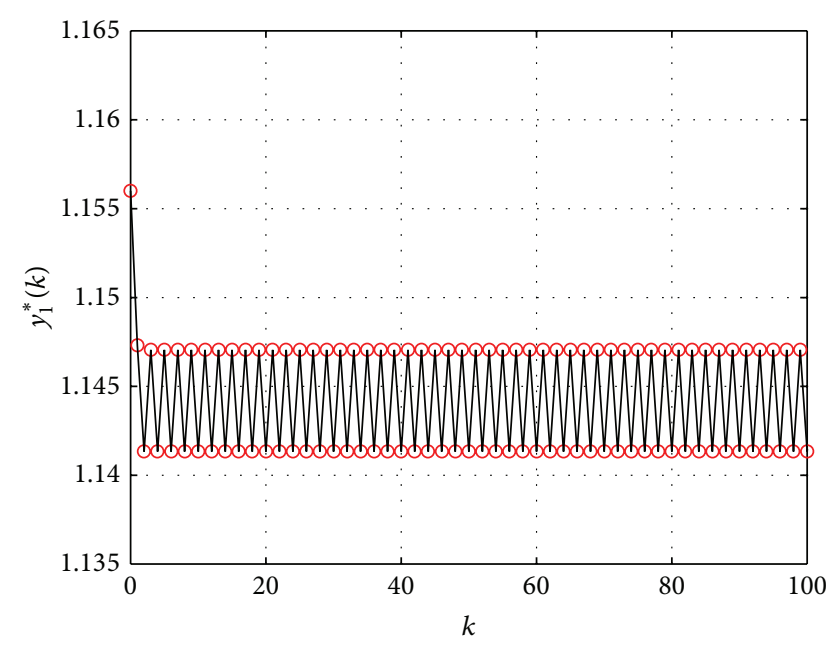

○ $y_{1}^{*}(k)$

(a)

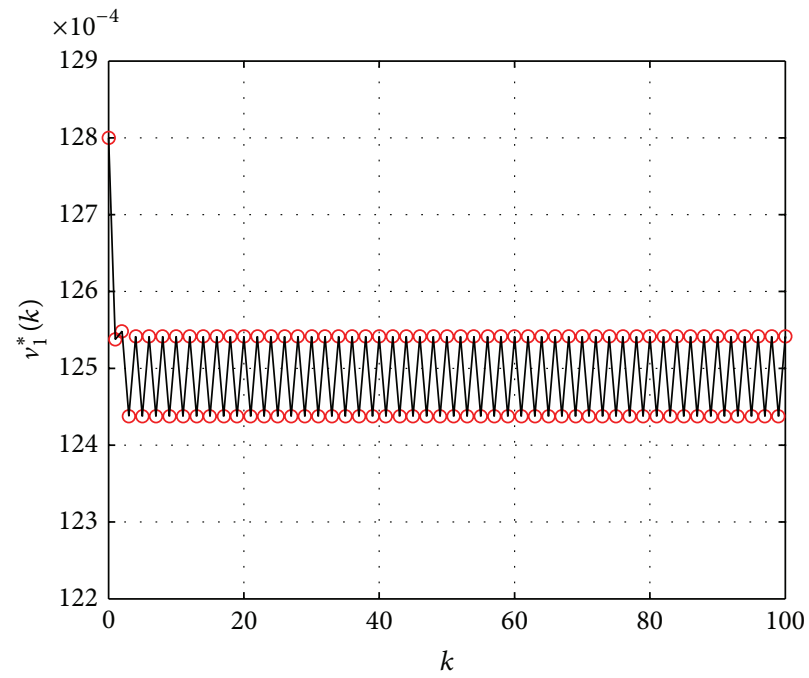

$\circ v_{1}^{*}(k)$

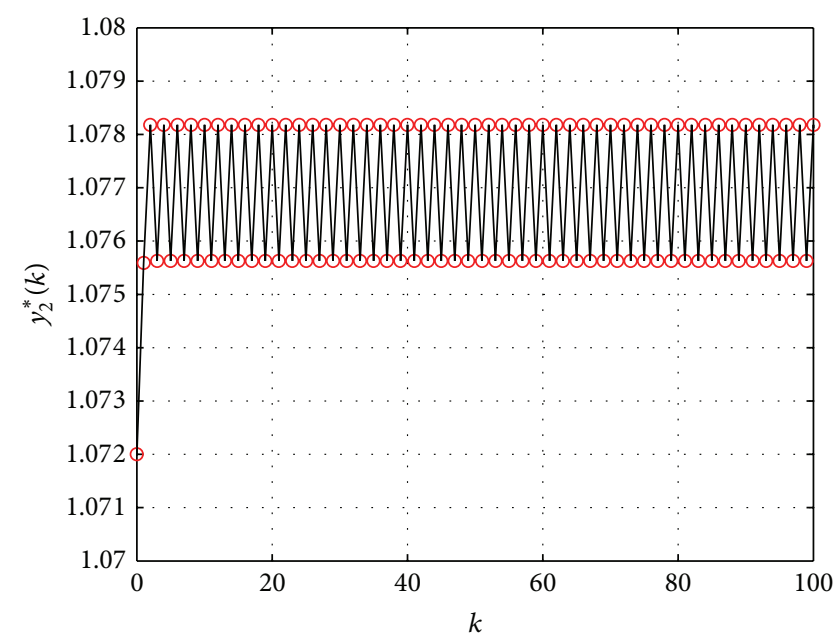

$\circ y_{2}^{*}(k)$

(b)

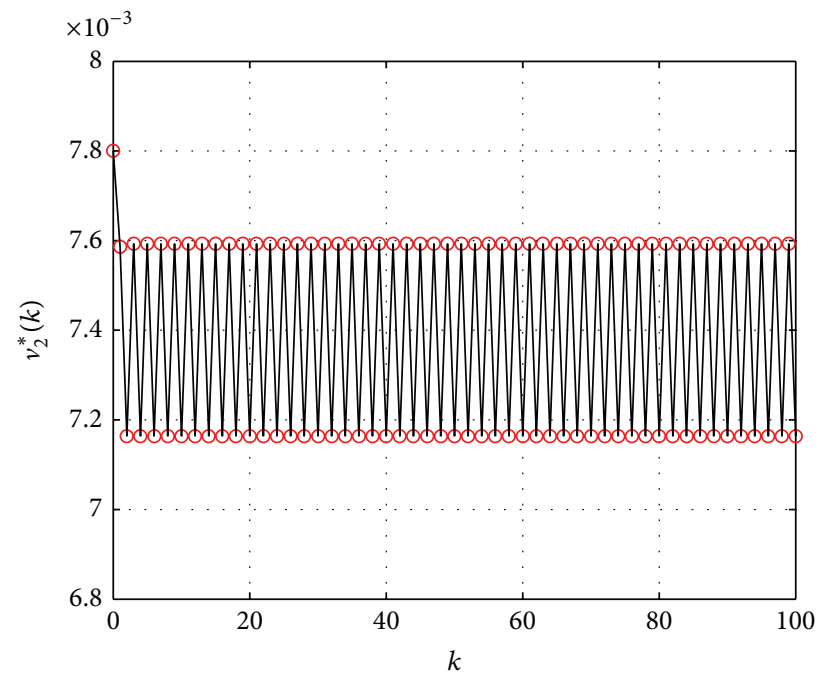

○ $v_{2}^{*}(k)$

(c)

(d)

FIGURE 1: Positive periodic solution of system (6). (a)-(d) Time-series of $y_{1}^{*}(k), y_{2}^{*}(k), v_{1}^{*}(k)$, and $v_{2}^{*}(k)$ with initial values $y_{1}^{*}(0)=1.1560$, $y_{2}^{*}(0)=1.0720, v_{1}^{*}(0)=0.0128$, and $v_{2}^{*}(0)=0.0078$ for $k \in[0,100]$, respectively.

Here we use the fact that $\max _{x \in \mathbb{R}^{+}}[x \exp (a-b x)]=[\exp (a-$ $1)] / b$ for $a, b>0$ and $\mathbb{R}^{+}$is the set of all positive real numbers. Thus $y_{1}\left(k_{0}\right) \leq y_{1}\left(k_{0}+1\right) \leq M_{1}$.

One claims that $y_{1}(k) \leq M_{1}$ for all $k \geq k_{0}$. Otherwise, if there is a $l_{0}>k_{0}$ such that $y_{1}\left(l_{0}\right)>M_{1}$, then $l_{0} \geq k_{0}+2$. Set

$$
\bar{l}_{0}=\min \left\{l_{0}: l_{0} \geq k_{0}+2, y_{1}\left(l_{0}\right)>M_{1}\right\} ;
$$

namely, $y_{1}\left(\bar{l}_{0}\right)>M_{1}$ and $\bar{l}_{0} \geq k_{0}+2$; then $y_{1}\left(\bar{l}_{0}\right)>M_{1} \geq$ $y_{1}\left(\bar{l}_{0}-1\right)$. From the above discussion one obtains that $y_{1}\left(\bar{l}_{0}\right) \leq$ $M_{1}$, which is a contradiction. Hence, $y_{1}(k) \leq M_{1}$ for all $k \geq$ $k_{0}$; then lim $\sup _{k \rightarrow+\infty} y_{1}(k) \leq M_{1}$. Case $I$ is complete.

Case II. Assume that $y_{1}(k)>y_{1}(k+1)$ for $k \in \mathbb{Z}^{+}$. $\lim _{k \rightarrow+\infty} y_{1}(k)$ especially exists, denoted by $\bar{y}_{1}$. One claims that $\bar{y}_{1} \leq a_{1}^{U} / b_{1}^{L}$. Otherwise, if $\bar{y}_{1}>a_{1}^{U} / b_{1}^{L}$, then it follows from the first equation of system (2) that

$$
\begin{array}{r}
\lim _{k \rightarrow+\infty}\left[a_{1}(k)-b_{1}(k) y_{1}(k)-c_{1}(k) y_{2}(k)\right. \\
\left.-d_{1}(k) y_{1}^{2}(k)-e_{1}(k) v_{1}(k)\right]=0,
\end{array}
$$

which is a contradiction since

$$
\begin{gathered}
\lim _{k \rightarrow+\infty}\left[a_{1}(k)-b_{1}(k) y_{1}(k)-c_{1}(k) y_{2}(k)\right. \\
\left.-d_{1}(k) y_{1}^{2}(k)-e_{1}(k) v_{1}(k)\right]
\end{gathered}
$$




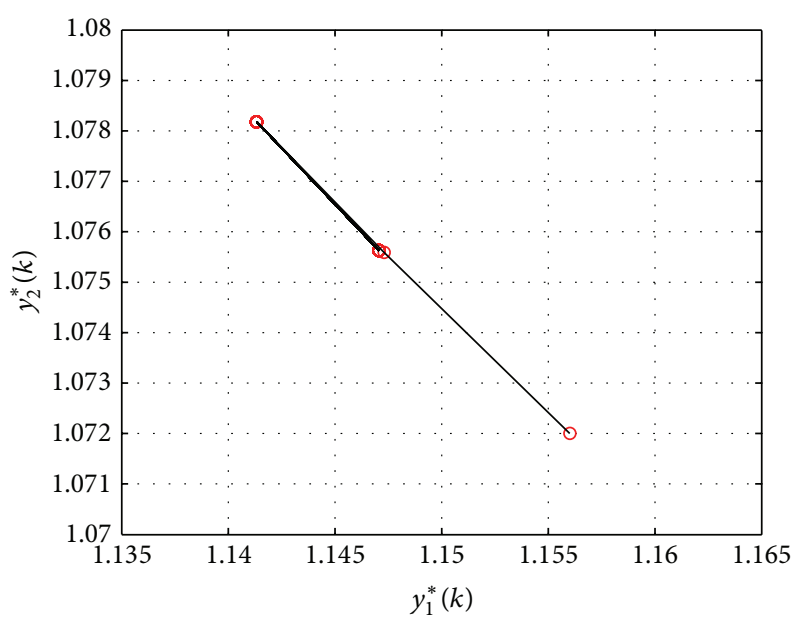

(a)

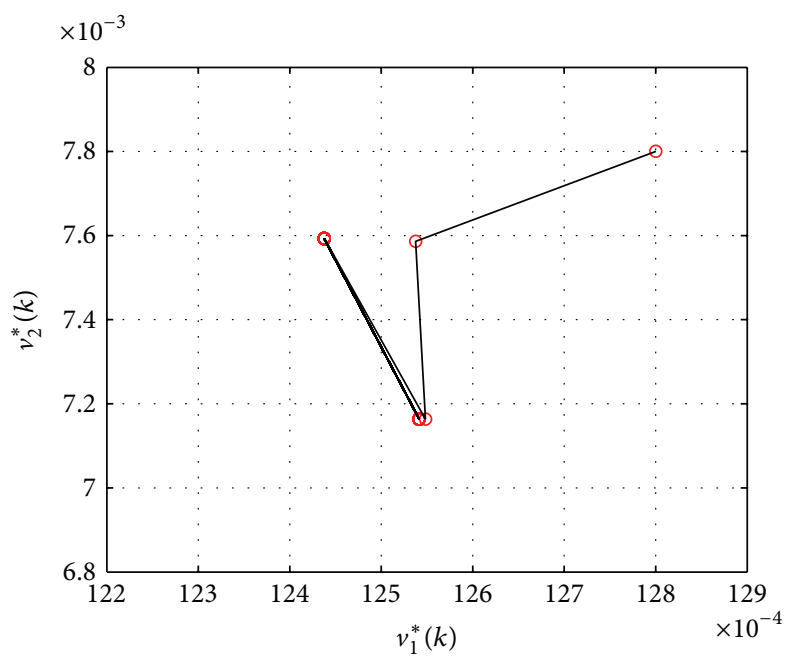

(b)

Figure 2: Two-dimensional phase portrait. (a) Phase portrait of $y_{1}^{*}(k)$ and $y_{2}^{*}(k)$ with initial values $y_{1}^{*}(0)=1.1560$ and $y_{2}^{*}(0)=1.0720$ for $k \in[0,100]$, respectively. (b) Phase portrait of $v_{1}^{*}(k)$ and $v_{2}^{*}(k)$ with initial values $v_{1}^{*}(0)=0.0128$ and $v_{2}^{*}(0)=0.0078$ for $k \in[0,100]$, respectively.

$$
\begin{aligned}
& \leq \lim _{k \rightarrow+\infty}\left[a_{1}(k)-b_{1}(k) y_{1}(k)\right] \\
& \leq a_{1}^{U}-b_{1}^{L} \bar{y}_{1}<0 .
\end{aligned}
$$

This proves the claim. By $\bar{y}_{1} \leq a_{1}^{U} / b_{1}^{L}$ and the fact that $\min _{x \in \mathbb{R}^{+}}[\exp (x-1) / x]=1$, one can show that

$$
\bar{y}_{1} \leq \frac{a_{1}^{U}}{b_{1}^{L}} \leq\left(\frac{a_{1}^{U}}{b_{1}^{L}}\right) \frac{\exp \left(a_{1}^{U}-1\right)}{a_{1}^{U}}=M_{1} .
$$

Hence $\lim \sup _{k \rightarrow+\infty} y_{1}(k) \leq M_{1}$. We can prove that $\lim \sup _{k \rightarrow+\infty} y_{2}(k) \leq M_{2}$ in the same way.

In the following, we prove that $\lim _{\sup _{k \rightarrow+\infty}} v_{1}(k) \leq N_{1}$. For any $\varepsilon>0$, there exists an integer $m_{0} \in \mathbb{Z}^{+}$such that $y_{1}(k) \leq M_{1}+\varepsilon$ for all $k \geq m_{0}$. We have from the third equation of system (2) that

$$
\begin{aligned}
v_{1}(k)= & \prod_{i=0}^{k-1}\left(1-f_{1}(i)\right)\left[v_{1}(0)+\sum_{i=0}^{k-1} \frac{h_{1}(i)+g_{1}(i) y_{1}(i)}{\prod_{j=0}^{i}\left(1-f_{1}(j)\right)}\right] \\
\leq & \left(1-f_{1}^{L}\right)^{k}\left(v_{1}(0)+\mu_{1}\right) \\
& +\left[h_{1}^{U}+g_{1}^{U}\left(M_{1}+\varepsilon\right)\right] \sum_{i=m_{0}}^{k-1} \prod_{j=i+1}^{k-1}\left(1-f_{1}(j)\right) \\
& \leq\left(1-f_{1}^{L}\right)^{k}\left(v_{1}(0)+\mu_{1}\right) \\
& +\left[h_{1}^{U}+g_{1}^{U}\left(M_{1}+\varepsilon\right)\right] \sum_{i=m_{0}}^{k-1}\left(1-f_{1}^{L}\right)^{k-i-1}
\end{aligned}
$$

where $\mu_{1}=\sum_{i=0}^{m_{0}-1}\left(\left(h_{1}(i)+g_{1}(i) y_{1}(i)\right) /\left(\prod_{j=0}^{i}\left(1-f_{1}(j)\right)\right)\right)$. Since $0<f_{1}^{L}<1$, we can choose a constant $\theta>0$ such that $1-f_{1}^{L}=e^{-\theta}$. By using the Stolz theorem, one has, as $k \rightarrow+\infty$,

$$
\sum_{i=m_{0}}^{k-1}\left(1-f_{1}^{L}\right)^{k-i-1}=\frac{\sum_{i=m_{0}}^{k-1} e^{\theta(i+1)}}{e^{\theta k}} \longrightarrow \frac{1}{1-e^{-\theta}}=\frac{1}{f_{1}^{L}}
$$

So $\lim \sup _{k \rightarrow+\infty} v_{1}(k) \leq\left(h_{1}^{U}+g_{1}^{U}\left(M_{1}+\varepsilon\right)\right) / f_{1}^{L}$. Since $\varepsilon$ is arbitrary, we obtain that $\lim \sup _{k \rightarrow+\infty} v_{1}(k) \leq N_{1}$.

Repeating a similar argument, we can prove that $\lim \sup _{k \rightarrow+\infty} v_{2}(k) \leq N_{2}$. The proof of Lemma 1 is complete.

\section{B. Proof of Lemma 2}

In view of Lemma 1 , we obtain that there exist $\varepsilon>0$ and $k^{*} \in$ $\mathbb{Z}^{+}$such that, for $k \geq k^{*}$,

$$
y_{i}(k) \leq M_{i}+\varepsilon, \quad v_{i}(k) \leq N_{i}+\varepsilon, \quad i=1,2 .
$$

The first inequality of (4) implies that

$$
a_{1}^{L}-c_{1}^{U}\left(M_{2}+\varepsilon\right)-d_{1}^{U}\left(M_{1}+\varepsilon\right)^{2}-e_{1}^{U}\left(N_{1}+\varepsilon\right)>0 .
$$

Similar to Lemma 1, we consider the following Cases I and II to show that

$$
\liminf _{k \rightarrow+\infty} y_{1}(k) \geq m_{1}
$$



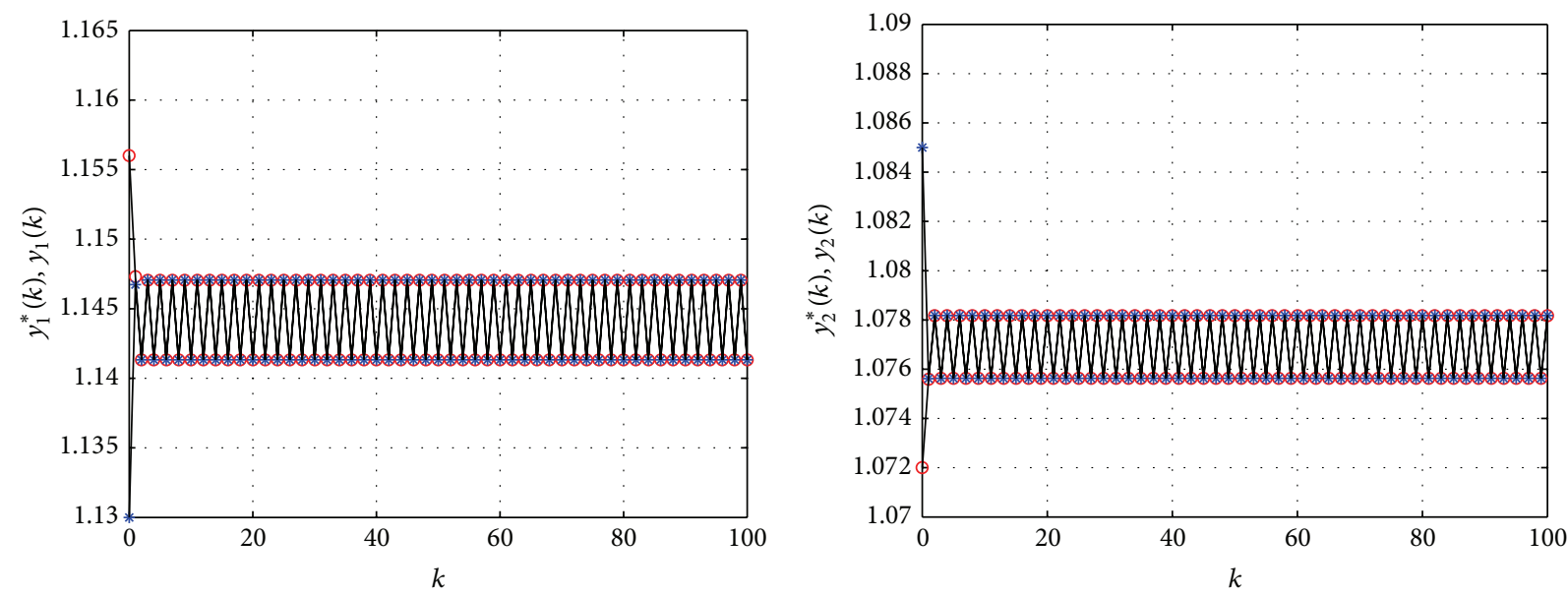
○ $y_{1}^{*}(k)$
○ $y_{2}^{*}(k)$
* $y_{1}(k)$
* $y_{2}(k)$

(a)
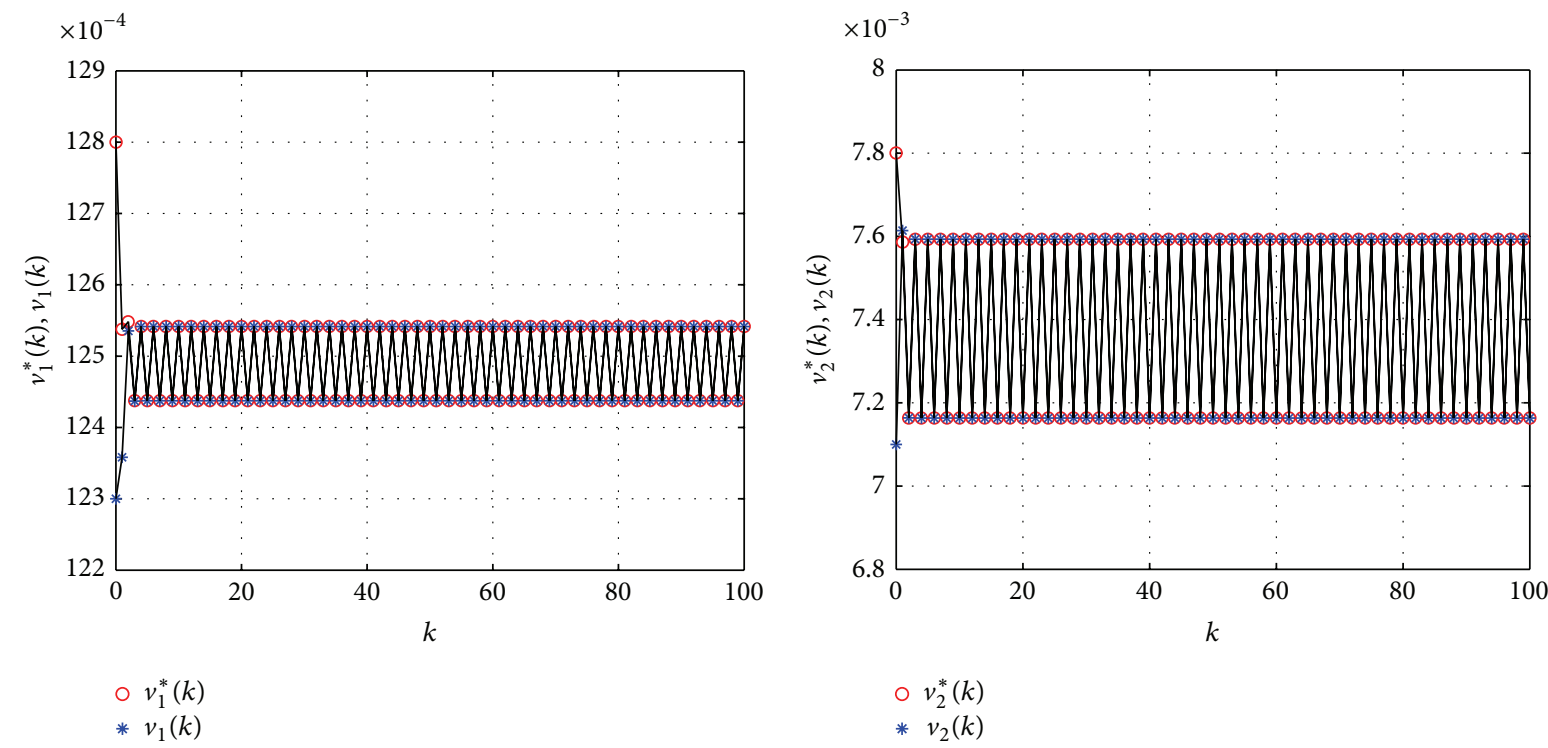

(c)

(d)

FIGURE 3: Global asymptotic stability. (a)-(d) Time-series of $y_{1}^{*}(k), y_{2}^{*}(k), v_{1}^{*}(k)$, and $v_{2}^{*}(k)$ with initial values $y_{1}^{*}(0)=1.1560, y_{2}^{*}(0)=1.0720$, $v_{1}^{*}(0)=0.0128$, and $v_{2}^{*}(0)=0.0078$ and $y_{1}(k), y_{2}(k), v_{1}(k)$, and $v_{2}(k)$ with initial values $y_{1}(0)=1.1300, y_{2}(0)=1.0850, v_{1}(0)=0.0123$, and $v_{2}(0)=0.0071$ for $k \in[0,100]$, respectively.

Case I. Suppose that there is a nonnegative integer $k_{0} \geq k^{*}$ such that $y_{1}\left(k_{0}+1\right) \leq y_{1}\left(k_{0}\right)$; we have from the first equation of system (2) that

$$
\begin{aligned}
y_{1}\left(k_{0}+1\right)=y_{1}\left(k_{0}\right) \exp [ & a_{1}\left(k_{0}\right)-b_{1}\left(k_{0}\right) y_{1}\left(k_{0}\right) \\
& -c_{1}\left(k_{0}\right) y_{2}\left(k_{0}\right)-d_{1}\left(k_{0}\right) y_{1}^{2}\left(k_{0}\right) \\
& \left.-e_{1}\left(k_{0}\right) v_{1}\left(k_{0}\right)\right] \\
\geq y_{1}\left(k_{0}\right) \exp [ & a_{1}^{L}-b_{1}^{U} y_{1}\left(k_{0}\right)-c_{1}^{U}\left(M_{2}+\varepsilon\right) \\
& \left.-d_{1}^{U}\left(M_{1}+\varepsilon\right)^{2}-e_{1}^{U}\left(N_{1}+\varepsilon\right)\right],
\end{aligned}
$$

and hence

$$
\begin{aligned}
& a_{1}^{L}-b_{1}^{U} y_{1}\left(k_{0}\right)-c_{1}^{U}\left(M_{2}+\varepsilon\right)-d_{1}^{U}\left(M_{1}+\varepsilon\right)^{2}-e_{1}^{U}\left(N_{1}+\varepsilon\right) \\
& \quad \leq 0 .
\end{aligned}
$$

Note that (B.2) and $\varepsilon$ is sufficiently small; one has from (B.5) that

$$
\begin{aligned}
y_{1}\left(k_{0}\right) & \geq \frac{a_{1}^{L}-c_{1}^{U}\left(M_{2}+\varepsilon\right)-d_{1}^{U}\left(M_{1}+\varepsilon\right)^{2}-e_{1}^{U}\left(N_{1}+\varepsilon\right)}{b_{1}^{U}} \\
& >0 .
\end{aligned}
$$




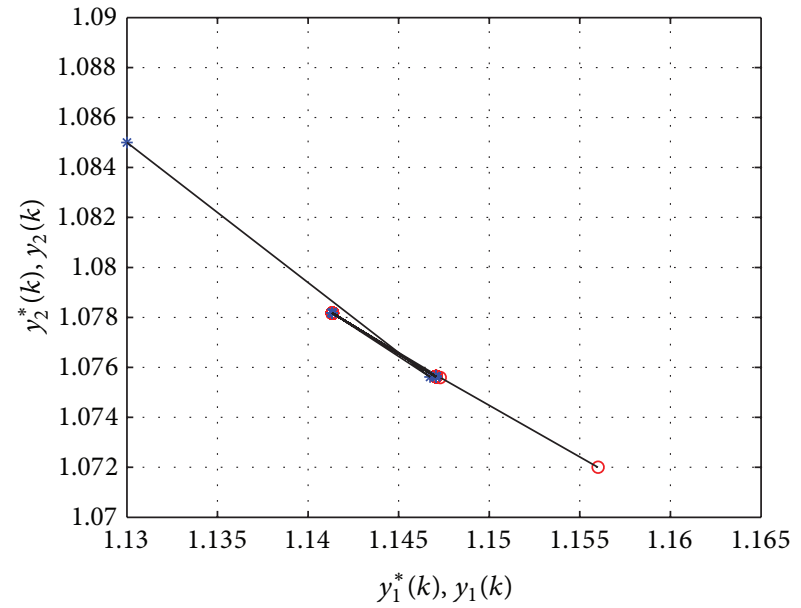

(a)

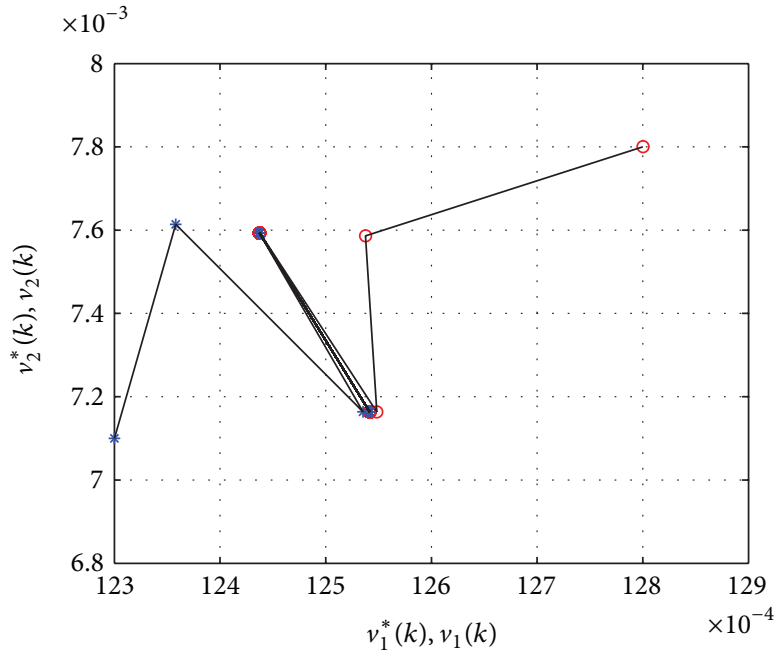

(b)

Figure 4: Two-dimensional phase portrait. (a) Phase portrait of $y_{1}^{*}(k)$ and $y_{2}^{*}(k)$ with initial values $y_{1}^{*}(0)=1.1560$ and $y_{2}^{*}(0)=1.0720$ and $y_{1}(k)$ and $y_{2}(k)$ with initial values $y_{1}(0)=1.1300$ and $y_{2}(0)=1.0850$ for $k \in[0,100]$, respectively. (b) Phase portrait of $v_{1}^{*}(k)$ and $v_{2}^{*}(k)$ with initial values $v_{1}^{*}(0)=0.0128$ and $v_{2}^{*}(0)=0.0078$ and $v_{1}(k)$ and $v_{2}(k)$ with initial values $v_{1}(0)=0.0123$ and $v_{2}(0)=0.0071$ for $k \in[0,100]$, respectively.

Furthermore, it follows from (B.4) and (B.6) that

$$
\begin{aligned}
& y_{1}\left(k_{0}+1\right) \\
& =y_{1}\left(k_{0}\right) \exp \left[a_{1}\left(k_{0}\right)-b_{1}\left(k_{0}\right) y_{1}\left(k_{0}\right)-c_{1}\left(k_{0}\right) y_{2}\left(k_{0}\right)\right. \\
& \left.-d_{1}\left(k_{0}\right) y_{1}^{2}\left(k_{0}\right)-e_{1}\left(k_{0}\right) v_{1}\left(k_{0}\right)\right] \\
& \geq \frac{a_{1}^{L}-c_{1}^{U}\left(M_{2}+\varepsilon\right)-d_{1}^{U}\left(M_{1}+\varepsilon\right)^{2}-e_{1}^{U}\left(N_{1}+\varepsilon\right)}{b_{1}^{U}} \\
& \quad \times \exp \left[a_{1}^{L}-b_{1}^{U}\left(M_{1}+\varepsilon\right)-c_{1}^{U}\left(M_{2}+\varepsilon\right)\right. \\
& \left.-d_{1}^{U}\left(M_{1}+\varepsilon\right)^{2}-e_{1}^{U}\left(N_{1}+\varepsilon\right)\right]
\end{aligned}
$$

$>0$.

Hence $y_{1}\left(k_{0}\right) \geq y_{1}\left(k_{0}+1\right) \geq y_{1}^{\varepsilon}$, where

$$
\begin{gathered}
y_{1}^{\varepsilon}=\frac{a_{1}^{L}-c_{1}^{U}\left(M_{2}+\varepsilon\right)-d_{1}^{U}\left(M_{1}+\varepsilon\right)^{2}-e_{1}^{U}\left(N_{1}+\varepsilon\right)}{b_{1}^{U}} \\
\times \exp \left[a_{1}^{L}-b_{1}^{U}\left(M_{1}+\varepsilon\right)-c_{1}^{U}\left(M_{2}+\varepsilon\right)\right. \\
\left.-d_{1}^{U}\left(M_{1}+\varepsilon\right)^{2}-e_{1}^{U}\left(N_{1}+\varepsilon\right)\right] .
\end{gathered}
$$

Now, we claim that $y_{1}(k) \geq y_{1}^{\varepsilon}$ for all $k \geq k_{0}$. Otherwise, if there exists a $q_{0}>k_{0}$ such that $y_{1}\left(q_{0}\right)<y_{1}^{\varepsilon}$, then $q_{0} \geq k_{0}+2$. Set

$$
\bar{q}_{0}=\min \left\{q_{0}: q_{0} \geq k_{0}+2, y_{1}\left(q_{0}\right)<y_{1}^{\varepsilon}\right\}
$$

then $y_{1}\left(\bar{q}_{0}-1\right)>y_{1}\left(\bar{q}_{0}\right)$. The above argument produces that $y_{1}\left(\bar{q}_{0}\right) \geq y_{1}^{\varepsilon}$, which is a contradiction. Thus $y_{1}(k) \geq y_{1}^{\varepsilon}$ for all $k \geq k_{0}$. Since $\varepsilon$ is sufficiently small, this reduces to

$$
\begin{aligned}
y_{1}(k) \geq & \frac{a_{1}^{L}-c_{1}^{U} M_{2}-d_{1}^{U} M_{1}^{2}-e_{1}^{U} N_{1}}{b_{1}^{U}} \\
& \times \exp \left[a_{1}^{L}-b_{1}^{U} M_{1}-c_{1}^{U} M_{2}-d_{1}^{U} M_{1}^{2}-e_{1}^{U} N_{1}\right]=m_{1} .
\end{aligned}
$$

Hence, $\liminf \operatorname{int+\infty }_{k \rightarrow+} y_{1}(k) \geq m_{1}$. This verifies Case $I$.

Case II. We assume that $y_{1}(k+1) \geq y_{1}(k)$ for all large $k \geq k^{*}$. In this case, $\lim _{k \rightarrow+\infty} y_{1}(k)$ exists, denoted by $y_{1 *}$. One claims that $y_{1 *} \geq\left(a_{1}^{L}-c_{1}^{U}\left(M_{2}+\varepsilon\right)-d_{1}^{U}\left(M_{1}+\varepsilon\right)^{2}-e_{1}^{U}\left(N_{1}+\varepsilon\right)\right) / b_{1}^{U}$; otherwise, assume that $y_{1 *}<\left(a_{1}^{L}-c_{1}^{U}\left(M_{2}+\varepsilon\right)-d_{1}^{U}\left(M_{1}+\varepsilon\right)^{2}-\right.$ $\left.e_{1}^{U}\left(N_{1}+\varepsilon\right)\right) / b_{1}^{U}$. It follows from the first equation of system (2) that

$$
\begin{array}{r}
\lim _{k \rightarrow+\infty}\left[a_{1}(k)-b_{1}(k) y_{1}(k)-c_{1}(k) y_{2}(k)\right. \\
\left.-d_{1}(k) y_{1}^{2}(k)-e_{1}(k) v_{1}(k)\right]=0 ;
\end{array}
$$

however,

$$
\begin{aligned}
\lim _{k \rightarrow+\infty}\left[a_{1}(k)-b_{1}(k) y_{1}(k)-c_{1}(k) y_{2}(k)\right. & \\
- & \left.d_{1}(k) y_{1}^{2}(k)-e_{1}(k) v_{1}(k)\right] \\
\geq \lim _{k \rightarrow+\infty}[ & {\left[a_{1}(k)-b_{1}(k) y_{1}(k)-c_{1}(k)\left(M_{2}+\varepsilon\right)\right.} \\
& \left.-d_{1}(k)\left(M_{1}+\varepsilon\right)^{2}-e_{1}(k)\left(N_{1}+\varepsilon\right)\right]
\end{aligned}
$$


$\geq a_{1}^{L}-b_{1}^{U} y_{1 *}-c_{1}^{U}\left(M_{2}+\varepsilon\right)-d_{1}^{U}\left(M_{1}+\varepsilon\right)^{2}-e_{1}^{U}\left(N_{1}+\varepsilon\right)$ $>0$,

which is a contradiction. This fact implies that $\lim \inf _{k \rightarrow+\infty} y_{1}(k) \geq y_{1}^{\varepsilon}$. Since $\varepsilon$ can be sufficiently small, one yields $\lim \inf _{k \rightarrow+\infty} y_{1}(k) \geq m_{1}$, where $m_{1}$ is defined as (B.10).

Similarly, one can derive that $\liminf _{k \rightarrow+\infty} y_{2}(k) \geq m_{2}$.

In the following, we prove that $\liminf _{k \rightarrow+\infty} v_{1}(k) \geq n_{1}$. For any $\varepsilon>0$, there exists an integer $p_{0} \in \mathbb{Z}^{+}$such that $y_{1}(k) \geq m_{1}-\varepsilon$ for $k \geq p_{0}$. We have from the third equation of system (2) that

$$
\begin{aligned}
v_{1}(k)= & \prod_{i=0}^{k-1}\left(1-f_{1}(i)\right)\left[v_{1}(0)+\sum_{i=0}^{k-1} \frac{h_{1}(i)+g_{1}(i) y_{1}(i)}{\prod_{j=0}^{i}\left(1-f_{1}(j)\right)}\right] \\
\geq & \left(1-f_{1}^{U}\right)^{k}\left(v_{1}(0)+\mu_{2}\right) \\
& +\left[h_{1}^{L}+g_{1}^{L}\left(m_{1}-\varepsilon\right)\right] \sum_{i=p_{0}}^{k-1} \prod_{j=i+1}^{k-1}\left(1-f_{1}(j)\right) \\
\geq & \left(1-f_{1}^{U}\right)^{k}\left(v_{1}(0)+\mu_{2}\right) \\
& +\left[h_{1}^{L}+g_{1}^{L}\left(m_{1}-\varepsilon\right)\right] \sum_{i=p_{0}}^{k-1}\left(1-f_{1}^{U}\right)^{k-i-1},
\end{aligned}
$$

where $\mu_{2}=\sum_{i=0}^{p_{0}-1}\left(\left(h_{1}(i)+g_{1}(i) y_{1}(i)\right) /\left(\prod_{j=0}^{i}\left(1-f_{1}(j)\right)\right)\right)$. Since $0<f_{1}^{U}<1$, we can choose a constant $\eta>0$ such that $1-f_{1}^{U}=$ $e^{-\eta} ;$ then by Stolz's theorem one yields

$$
\begin{array}{r}
\sum_{i=p_{0}}^{k-1}\left(1-f_{1}^{U}\right)^{k-i-1}=\frac{\sum_{i=p_{0}}^{k-1} e^{\eta(i+1)}}{e^{\eta k}} \longrightarrow \frac{1}{1-e^{-\eta}}=\frac{1}{f_{1}^{U}} \\
\text { as } k \longrightarrow+\infty .
\end{array}
$$

Then $\lim \inf _{k \rightarrow+\infty} v_{1}(k) \geq\left(h_{1}^{L}+g_{1}^{L}\left(m_{1}-\varepsilon\right)\right) / f_{1}^{U}$. Since $\varepsilon$ can be sufficiently small, we have

$$
\liminf _{k \rightarrow+\infty} v_{1}(k) \geq n_{1}=\frac{\left(h_{1}^{L}+g_{1}^{L} m_{1}\right)}{f_{1}^{U}} .
$$

Analogously,

$$
\liminf _{k \rightarrow+\infty} v_{2}(k) \geq n_{2}=\frac{\left(h_{2}^{L}+g_{2}^{L} m_{2}\right)}{f_{2}^{U}} .
$$

The proof of Lemma 2 is complete.

\section{Proof of Theorem 3}

It follows from Lemmas 1 and 2 that

$$
\Theta=\left[m_{1}, M_{1}\right] \times\left[m_{2}, M_{2}\right] \times\left[n_{1}, N_{1}\right] \times\left[n_{2}, N_{2}\right]
$$

is an invariant set of system (2). Define the continuous mapping $\Pi$ on $\Theta$ by

$$
\begin{aligned}
\Pi & \left\{y_{1}^{*}(0), y_{2}^{*}(0), v_{1}^{*}(0), v_{2}^{*}(0)\right\} \\
& =\Pi\left\{y_{1}^{*}(T), y_{2}^{*}(T), v_{1}^{*}(T), v_{2}^{*}(T)\right\},
\end{aligned}
$$

for $\left\{y_{1}^{*}(0), y_{2}^{*}(0), v_{1}^{*}(0), v_{2}^{*}(0)\right\} \in \Theta$. Clearly, $\Pi$ depends continuously on $\left\{y_{1}^{*}(0), y_{2}^{*}(0), v_{1}^{*}(0), v_{2}^{*}(0)\right\}$; then $\Pi$ is continuous and maps the compact set $\Theta$ into itself. Hence, $\Pi$ has a fixed point $\left\{\widetilde{y}_{1}, \widetilde{y}_{2}, \widetilde{v}_{1}, \widetilde{v}_{2}\right\}$. It is easy to see that the solution $\left\{y_{1}^{*}(k), y_{2}^{*}(k), v_{1}^{*}(k), v_{2}^{*}(k)\right\}$ which passes through $\left\{\widetilde{y}_{1}, \widetilde{y}_{2}, \widetilde{v}_{1}, \widetilde{v}_{2}\right\}$ is a $T$-periodic solution of system (2). The proof of Theorem 3 is complete.

\section{Proof of Theorem 4}

Let $\left\{y_{1}^{*}(k), y_{2}^{*}(k), v_{1}^{*}(k), v_{2}^{*}(k)\right\}$ be positive periodic solution and let $\left\{y_{1}(k), y_{2}(k), v_{1}(k), v_{2}(k)\right\}$ be any positive solution of system (2), respectively. Let us make the change of variables as follows:

$$
\begin{gathered}
x_{1}(k)=\ln y_{1}(k)-\ln y_{1}^{*}(k), \\
x_{2}(k)=\ln y_{2}(k)-\ln y_{2}^{*}(k), \\
u_{1}(k)=v_{1}(k)-v_{1}^{*}(k), \quad u_{2}(k)=v_{2}(k)-v_{2}^{*}(k) .
\end{gathered}
$$

By the mean-value theorem, we have from the first equation of system (2) that

$$
\begin{aligned}
x_{1}(k+1)= & \ln y_{1}(k+1)-\ln y_{1}^{*}(k+1) \\
= & \ln y_{1}(k)-\ln y_{1}^{*}(k)-b_{1}(k)\left(y_{1}(k)-y_{1}^{*}(k)\right) \\
& -c_{1}(k)\left(y_{2}(k)-y_{2}^{*}(k)\right) \\
& -d_{1}(k)\left(y_{1}^{2}(k)-y_{1}^{* 2}(k)\right) \\
& -e_{1}(k)\left(v_{1}(k)-v_{1}^{*}(k)\right) \\
= & \ln y_{1}(k)-\ln y_{1}^{*}(k)-b_{1}(k) \exp \left(\theta_{1}(k)\right) \\
& \times\left(\ln y_{1}(k)-\ln y_{1}^{*}(k)\right)-c_{1}(k) \exp \left(\theta_{2}(k)\right) \\
& \cdot\left(\ln y_{2}(k)-\ln y_{2}^{*}(k)\right)-2 d_{1}(k) \exp \left(2 \theta_{3}(k)\right) \\
& \times\left(\ln y_{1}(k)-\ln y_{1}^{*}(k)\right) \\
& -e_{1}(k)\left(v_{1}(k)-v_{1}^{*}(k)\right) \\
= & {\left[1-b_{1}(k) \exp \left(\theta_{1}(k)\right)\right.} \\
& \left.-2 d_{1}(k) \exp \left(2 \theta_{3}(k)\right)\right] x_{1}(k) \\
& -c_{1}(k) \exp \left(\theta_{2}(k)\right) x_{2}(k)-e_{1}(k) u_{1}(k) .
\end{aligned}
$$


Similarly, we can arrive at the following desired results:

$$
\begin{aligned}
& x_{2}(k+1)=\left[1-b_{2}(k) \exp \left(\theta_{4}(k)\right)\right. \\
& \left.-2 d_{2}(k) \exp \left(2 \theta_{6}(k)\right)\right] x_{2}(k) \\
& -c_{2}(k) \exp \left(\theta_{5}(k)\right) x_{1}(k)-e_{2}(k) u_{2}(k), \\
& u_{1}(k+1)=\left(1-f_{1}(k)\right) u_{1}(k)+g_{1}(k) \exp \left(\theta_{7}(k)\right) x_{1}(k) \text {, } \\
& u_{2}(k+1)=\left(1-f_{2}(k)\right) u_{2}(k)+g_{2}(k) \exp \left(\theta_{8}(k)\right) x_{2}(k) \text {, }
\end{aligned}
$$

where $\theta_{1}(k), \theta_{3}(k), \theta_{5}(k)$, and $\theta_{7}(k)$ lie between $\ln y_{1}^{*}(k)$ and $\ln y_{1}(k)$ and $\theta_{2}(k), \theta_{4}(k), \theta_{6}(k)$, and $\theta_{8}(k)$ lie between $\ln y_{2}^{*}(k)$ and $\ln y_{2}(k)$.

By the inequalities in (5), we will now select a constant $\varepsilon$ small enough such that

$$
\begin{gathered}
\rho_{1}^{\varepsilon}=\max \left\{\left|1-b_{1}^{L}\left(m_{1}-\varepsilon\right)-2 d_{1}^{L}\left(m_{1}-\varepsilon\right)^{2}\right|,\right. \\
\left.\left|1-b_{1}^{U}\left(M_{1}+\varepsilon\right)-2 d_{1}^{U}\left(M_{1}+\varepsilon\right)^{2}\right|\right\} \\
+c_{1}^{U}\left(M_{2}+\varepsilon\right)+e_{1}^{U}<1, \\
\rho_{2}^{\varepsilon}=\max \left\{\left|1-b_{2}^{L}\left(m_{2}-\varepsilon\right)-2 d_{2}^{L}\left(m_{2}-\varepsilon\right)^{2}\right|,\right. \\
\left.\quad\left|1-b_{2}^{U}\left(M_{2}+\varepsilon\right)-2 d_{2}^{U}\left(M_{2}+\varepsilon\right)^{2}\right|\right\} \\
+c_{2}^{U}\left(M_{1}+\varepsilon\right)+e_{2}^{U}<1, \\
\rho_{3}^{\varepsilon}=1-f_{1}^{L}+g_{1}^{U}\left(M_{1}+\varepsilon\right)<1, \\
\rho_{4}^{\varepsilon}=1-f_{2}^{L}+g_{2}^{U}\left(M_{2}+\varepsilon\right)<1 .
\end{gathered}
$$

From Lemmas 1 and 2 , we know that there exists a $\widehat{k} \in \mathbb{Z}^{+}$ such that, for $k \geq \widehat{k}$,

$$
\begin{aligned}
& 0<m_{1}-\varepsilon \leq y_{1}^{*}(k) \leq M_{1}+\varepsilon, \\
& 0<m_{1}-\varepsilon \leq y_{1}(k) \leq M_{1}+\varepsilon, \\
& 0<m_{2}-\varepsilon \leq y_{2}^{*}(k) \leq M_{2}+\varepsilon, \\
& 0<m_{2}-\varepsilon \leq y_{2}(k) \leq M_{2}+\varepsilon, \\
& 0<n_{1}-\varepsilon \leq v_{1}^{*}(k) \leq N_{1}+\varepsilon, \\
& 0<n_{1}-\varepsilon \leq v_{1}(k) \leq N_{1}+\varepsilon, \\
& 0<n_{2}-\varepsilon \leq v_{2}^{*}(k) \leq N_{2}+\varepsilon, \\
& 0<n_{2}-\varepsilon \leq v_{2}(k) \leq N_{2}+\varepsilon .
\end{aligned}
$$

It is easy to see that $\exp \left(\theta_{1}(k)\right), \exp \left(\theta_{3}(k)\right), \exp \left(\theta_{5}(k)\right)$, and $\exp \left(\theta_{7}(k)\right)$ are between $y_{1}^{*}(k)$ and $y_{1}(k)$. Meanwhile, $\exp \left(\theta_{2}(k)\right), \exp \left(\theta_{4}(k)\right), \exp \left(\theta_{6}(k)\right)$, and $\exp \left(\theta_{8}(k)\right)$ are between $y_{2}^{*}(k)$ and $y_{2}(k)$. It follows from the equation of (D.2) that

$$
\begin{aligned}
\left|x_{1}(k+1)\right|= & \left|1-b_{1}(k) \exp \left(\theta_{1}(k)\right)-2 d_{1}(k) \exp \left(2 \theta_{3}(k)\right)\right| \\
& \cdot\left|x_{1}(k)\right|+\left|c_{1}(k) \exp \left(\theta_{2}(k)\right)\right| \cdot\left|x_{2}(k)\right| \\
& +\left|e_{1}(k)\right| \cdot\left|u_{1}(k)\right| \\
\leq & \max \left\{\left|1-b_{1}^{L}\left(m_{1}-\varepsilon\right)-2 d_{1}^{L}\left(m_{1}-\varepsilon\right)^{2}\right|,\right. \\
& \left.\quad\left|1-b_{1}^{U}\left(M_{1}+\varepsilon\right)-2 d_{1}^{U}\left(M_{1}+\varepsilon\right)^{2}\right|\right\} \\
\leq & \rho_{1}^{\varepsilon} \max \left\{\left|x_{1}(k)\right|+c_{1}^{U}\left(M_{2}+\varepsilon\right)\left|x_{2}(k)\right|+e_{1}^{U}\left|u_{1}(k)\right|,\left|u_{1}(k)\right|,\left|u_{2}(k)\right|\right\} .
\end{aligned}
$$

Analogously,

$$
\begin{aligned}
& \left|x_{2}(k+1)\right| \leq \rho_{2}^{\varepsilon} \max \left\{\left|x_{1}(k)\right|,\left|x_{2}(k)\right|,\left|u_{1}(k)\right|,\left|u_{2}(k)\right|\right\}, \\
& \left|u_{1}(k+1)\right| \leq \rho_{3}^{\varepsilon} \max \left\{\left|x_{1}(k)\right|,\left|x_{2}(k)\right|,\left|u_{1}(k)\right|,\left|u_{2}(k)\right|\right\}, \\
& \left|u_{2}(k+1)\right| \leq \rho_{4}^{\varepsilon} \max \left\{\left|x_{1}(k)\right|,\left|x_{2}(k)\right|,\left|u_{1}(k)\right|,\left|u_{2}(k)\right|\right\} .
\end{aligned}
$$

Let $\rho^{\varepsilon}=\max \left\{\rho_{1}^{\varepsilon}, \rho_{2}^{\varepsilon}, \rho_{3}^{\varepsilon}, \rho_{4}^{\varepsilon}\right\}$; then $\rho^{\varepsilon}<1$. Hence, when $k \geq \widehat{k}$ we have

$$
\begin{aligned}
& \max \left\{\left|x_{1}(k+1)\right|,\left|x_{2}(k+1)\right|,\left|u_{1}(k+1)\right|,\left|u_{2}(k+1)\right|\right\} \\
& \leq \rho^{\varepsilon} \max \left\{\left|x_{1}(k)\right|,\left|x_{2}(k)\right|,\left|u_{1}(k)\right|,\left|u_{2}(k)\right|\right\} \\
& \quad \leq\left(\rho^{\varepsilon}\right)^{k-\widehat{k}} \max \left\{\left|x_{1}(\widehat{k})\right|,\left|x_{2}(\widehat{k})\right|,\left|u_{1}(\widehat{k})\right|,\left|u_{2}(\widehat{k})\right|\right\},
\end{aligned}
$$

which implies

$$
\begin{array}{ll}
\lim _{k \rightarrow+\infty}\left|y_{1}(k)-y_{1}^{*}(k)\right|=0, & \lim _{k \rightarrow+\infty}\left|y_{2}(k)-y_{2}^{*}(k)\right|=0, \\
\lim _{k \rightarrow+\infty}\left|v_{1}(k)-v_{1}^{*}(k)\right|=0, & \lim _{k \rightarrow+\infty}\left|v_{2}(k)-v_{2}^{*}(k)\right|=0 .
\end{array}
$$

So the positive periodic solution $\left\{y_{1}^{*}(k), y_{2}^{*}(k), v_{1}^{*}(k), v_{2}^{*}(k)\right\}$ of system (2) is globally asymptotically stable. The proof of Theorem 4 is complete.

\section{Conflict of Interests}

The authors declare that there is no conflict of interests regarding the publication of this paper.

\section{Acknowledgments}

The authors would like to thank the referees for their valuable comments and suggestions that greatly improved the presentation of this work. The work is supported by the National Natural Science Foundation of China (no. 11261017), 
the Key Project of Chinese Ministry of Education (no. 212111), the Key Laboratory of Biological Resources Protection and Utilization of Hubei Province (PKLHB1329), and the Key Subject of Hubei Province (Forestry).

\section{References}

[1] G. P. Samanta, "A two-species competitive system under the influence of toxic substances," Applied Mathematics and Computation, vol. 216, no. 1, pp. 291-299, 2010.

[2] J. D. Zhao, Z. C. Zhang, and J. Ju, "Necessary and sufficient conditions for permanence and extinction in a three dimensional competitive Lotka-Volterra system," Applied Mathematics and Computation, vol. 230, pp. 587-596, 2014.

[3] Z. J. Liu, M. Fan, and L. S. Chen, "Globally asymptotic stability in two periodic delayed competitive systems," Applied Mathematics and Computation, vol. 197, no. 1, pp. 271-287, 2008.

[4] X. Y. Song and L. S. Chen, "Periodic solution of a delay differential equation of plankton allelopathy," Acta Mathematica Scientia A, vol. 23, no. 1, pp. 8-13, 2003.

[5] X. Tang, D. Cao, and X. Zou, "Global attractivity of positive periodic solution to periodic Lotka-Volterra competition systems with pure delay," Journal of Differential Equations, vol. 228, no. 2, pp. 580-610, 2006.

[6] K. Gopalsamy, Stability and Oscillations in Delay Differential Equation of Population Dynamics, vol. 74 of Mathematics and Its Applications, Kluwer Academic Publishers, Dordrecht, The Netherlands, 1992.

[7] R. H. Tan, W. F. Liu, Q. L. Wang, and Z. J. Liu, "Uniformly asymptotic stability of almost periodic solutions for a competitive system with impulsive perturbations," Advances in Difference Equations, vol. 2014, article 2, 2014.

[8] Z. Liu, "Persistence and periodic solution in two species competitive system with feedback controls," Journal of Biomathematics, vol. 17, no. 2, pp. 251-255, 2002.

[9] M. Fan, K. Wang, P. J. Y. Wong, and R. P. Agarwal, "Periodicity and stability in periodic $n$-species Lotka-Volterra competition system with feedback controls and deviating arguments," Acta Mathematica Sinica (English Series), vol. 19, no. 4, pp. 801-822, 2003.

[10] F. D. Chen, "The permanence and global attractivity of LotkaVolterra competition system with feedback controls," Nonlinear Analysis: Real World Applications, vol. 7, no. 1, pp. 133-143, 2006.

[11] C. L. Shi, Z. Li, and F. D. Chen, "Extinction in a nonautonomous Lotka-Volterra competitive system with infinite delay and feedback controls," Nonlinear Analysis: Real World Applications, vol. 13, no. 5, pp. 2214-2226, 2012.

[12] Z. Li, M. Han, and F. Chen, "Influence of feedback controls on an autonomous Lotka-Volterra competitive system with infinite delays," Nonlinear Analysis: Real World Applications, vol. 14, no. 1, pp. 402-413, 2013.

[13] W. Yuan and C. Zhu, "Robust sampled-data $H_{\infty}$ output feedback control of active suspension system," International Journal of Innovative Computing, Information and Control, vol. 10, no. 1, pp. 281-292, 2014.

[14] S. Saat, D. Huang, and S. K. Nguang, "Robust state feedback control of uncertain polynomial discrete-time systems: an integral action approach," International Journal of Innovative Computing, Information and Control, vol. 9, no. 3, pp. 1233-1244, 2013. 


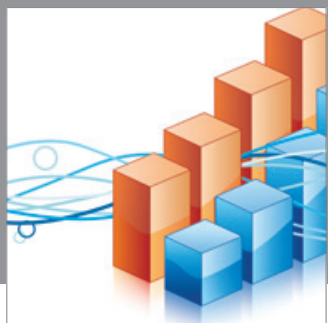

Advances in

Operations Research

mansans

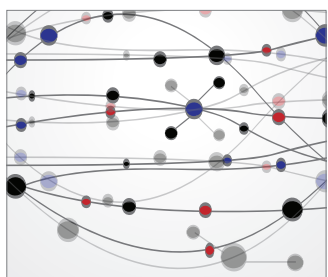

The Scientific World Journal
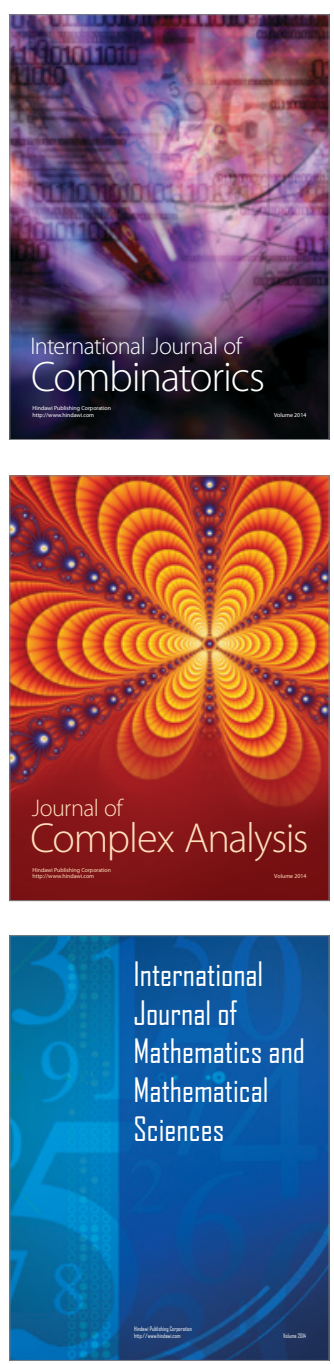
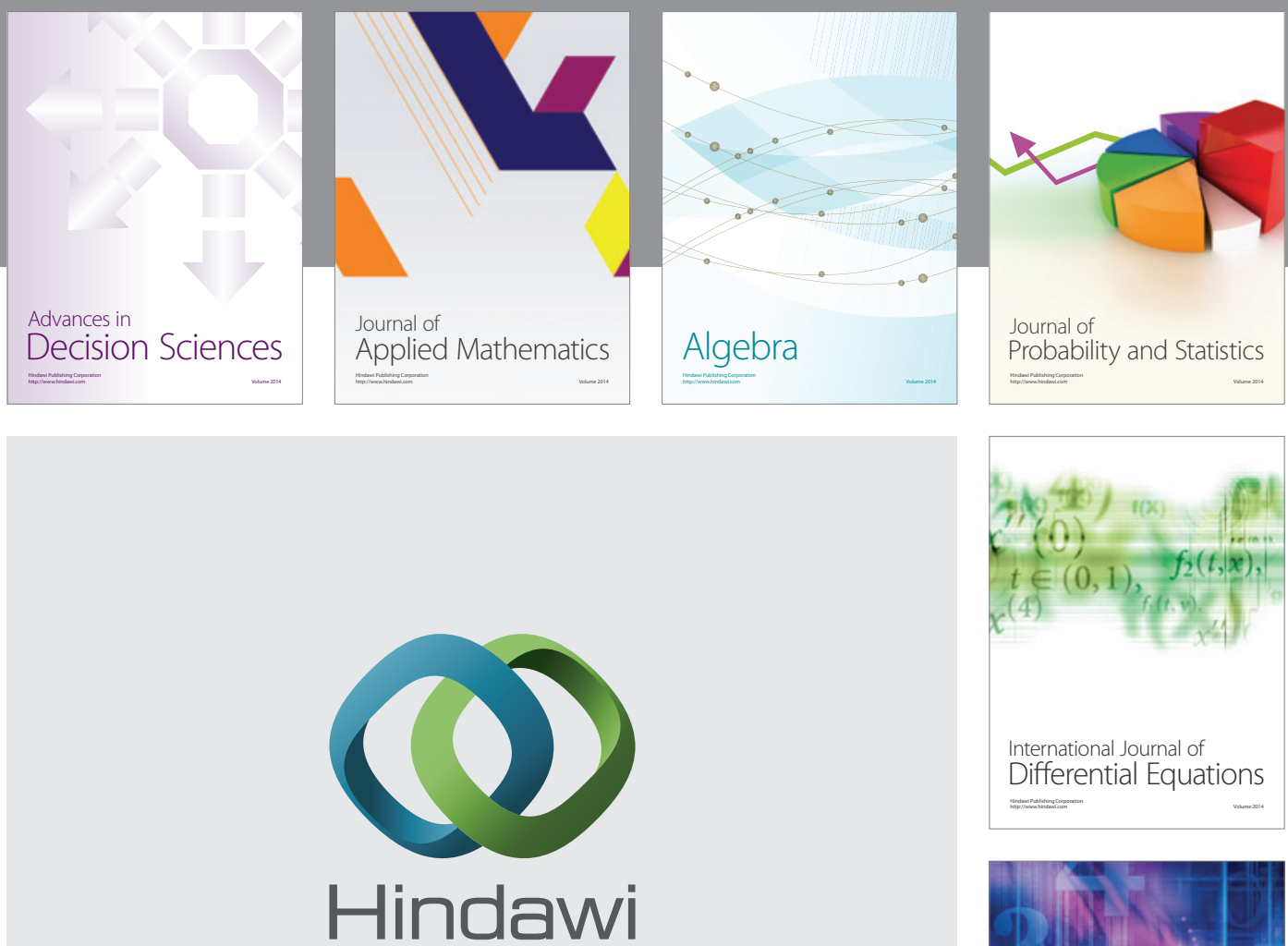

Submit your manuscripts at http://www.hindawi.com
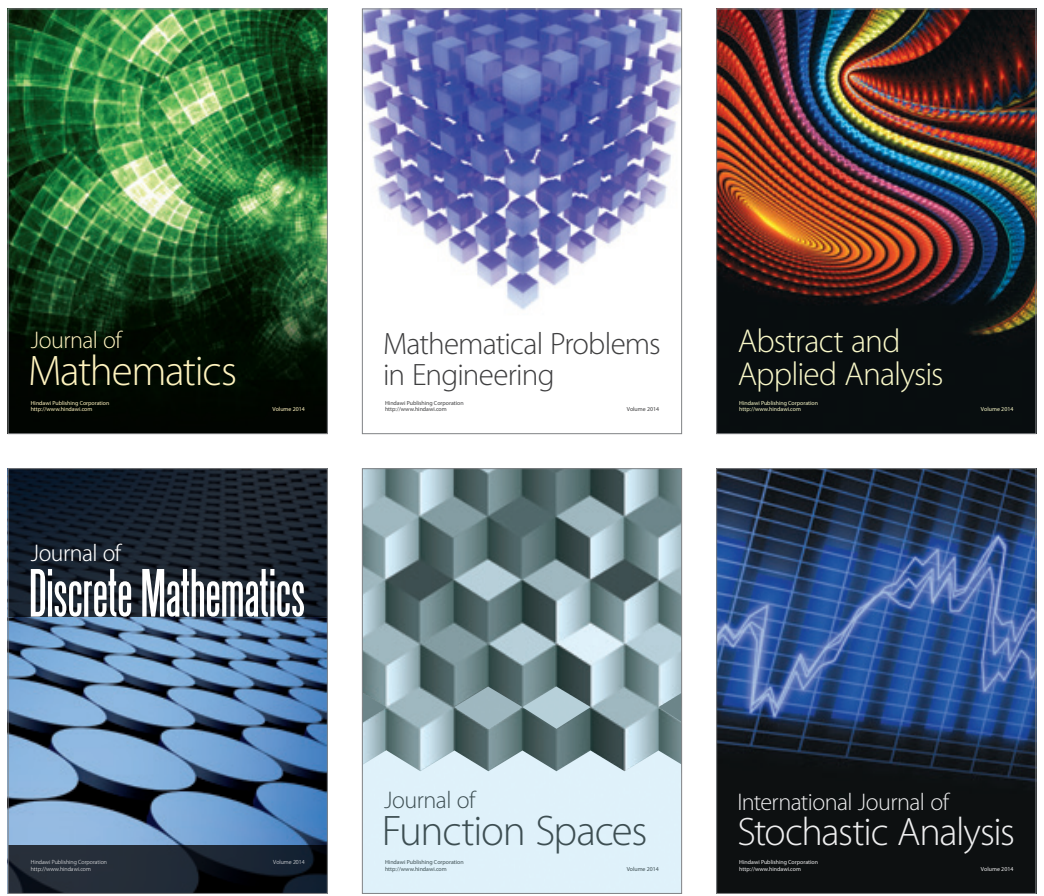

Journal of

Function Spaces

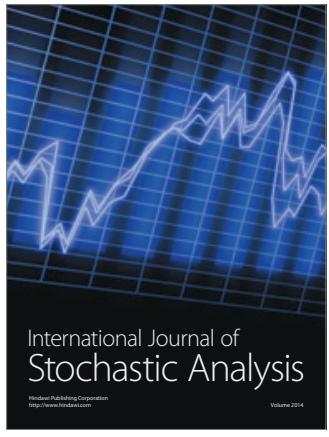

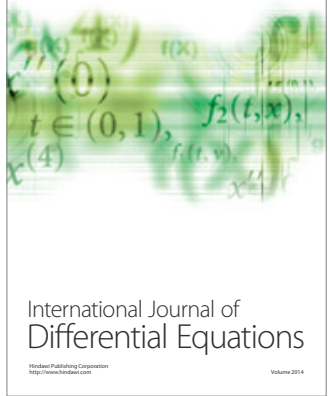
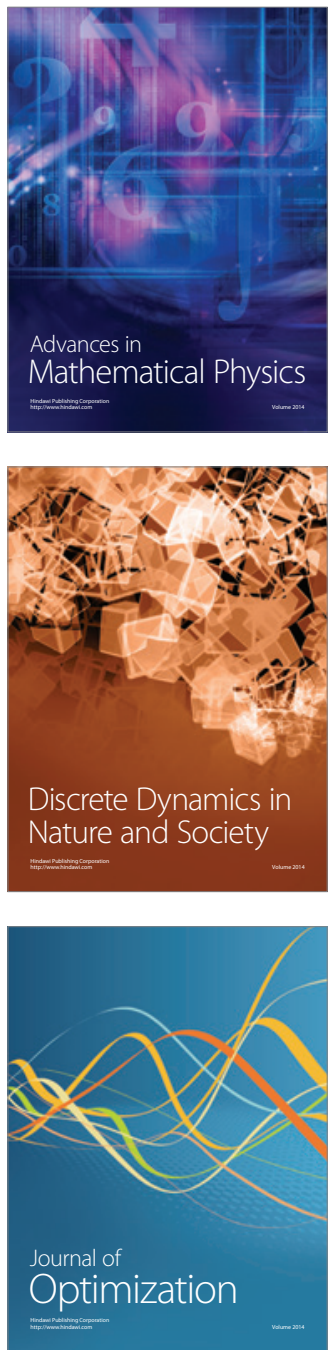